\title{
MOUNTAIN IMPASSE THEOREM AND SPECTRUM OF SEMILINEAR ELLIPTIC PROBLEMS
}

\author{
KYRIL TINTAREV
}

\begin{abstract}
This paper studies a minimax problem for functionals in Hilbert space in the form of $G(u)=\frac{1}{2} \rho\|u\|^{2}-g(u)$, where $g(u)$ is Fréchet differentiable with weakly continuous derivative. If $G$ has a "mountain pass geometry" it does not necessarily have a critical point. Such a case is called, in this paper, a "mountain impasse". This paper states that in a case of mountain impasse, there exists a sequence $u_{j} \in H$ such that
\end{abstract}

$$
g^{\prime}\left(u_{j}\right)=\rho_{j} u_{j}, \quad \rho_{j} \rightarrow \rho,\left\|u_{j}\right\| \rightarrow \infty,
$$

and $G\left(u_{j}\right)$ approximates the minimax value from above. If

$$
\gamma(t)=\sup _{\|u\|^{2}=t} g(u)
$$

and

$$
J_{0}=\left(2 \inf _{t_{2}>t_{1}>0} \frac{\gamma\left(t_{2}\right)-\gamma\left(t_{1}\right)}{t_{2}-t_{1}}, 2 \sup _{t_{2}>t_{1}>0} \frac{\gamma\left(t_{2}\right)-\gamma\left(t_{1}\right)}{t_{2}-t_{1}}\right),
$$

then $g^{\prime}(u)=\rho u$ has a nonzero solution $u$ for a dense subset of $\rho \in J_{0}$.

\section{FORMULATION OF RESULTS}

If $g$ is a $C^{1}$-functional on a Hilbert space and $u$ is a critical point of $g$ on a sphere, then $g^{\prime}(u)=\rho u, \rho \in \mathbf{R}$. This approach to semilinear elliptic equations has been known for decades (cf. [1,2]), but a question of the range of $\rho$ has remained open. A recent series of papers (cf. [7] and references therein) provides an answer that can be summarized as follows.

Let $H$ be an infinite dimensional Hilbert space and let $g: H \rightarrow \mathbf{R}$ be a $C^{1}$ map (with respect to Fréchet differentiation). Let $H_{w}$ be the space $H$ supplied with the weak topology. Assume that

$$
\begin{aligned}
& g \in C\left(H_{w} \rightarrow \mathbf{R}\right), \\
& g^{\prime} \in C\left(H_{w} \rightarrow H\right) .
\end{aligned}
$$

(By continuity we always mean local contiuity without uniform bounds.)

In applications to semilinear elliptic problems condition (1.1)-(1.2) correspond to the subcritical growth of the right-hand side.

Consider the following function

$$
\gamma(t)=\sup _{\|u\|^{2}=t} g(u) .
$$

Received by the editors December 3, 1990.

1980 Mathematics Subject Classification (1985 Revision). Primary 35P30, 35J65, 47H12. 
Theorem 1.1. Assume (1.1), (1.2). The function (1.3) is continuous, nondecreasing and possesses left- and right-hand derivatives $\gamma_{-}^{\prime}(t) \leq \gamma_{+}^{\prime}(t)$. For every $t>0$ such that $\gamma_{+}^{\prime}(t) \neq 0 \quad\left(\gamma_{-}^{\prime}(t) \neq 0\right)$ there exist $u_{+} \in H\left(u_{-} \in H\right)$ such that

$$
\left\|u_{ \pm}\right\|^{2}=t, \quad g\left(u_{ \pm}\right)=\gamma(t),
$$

and

$$
2 \gamma_{ \pm}^{\prime}(t) u_{ \pm}=g^{\prime}\left(u_{ \pm}\right) .
$$

In other words, the spectrum $\{\rho\}$ of the problem

$$
\rho u=g^{\prime}(u), \quad u \in H \backslash\{0\},
$$

contains all the tangent slopes from the graph of $2 \gamma(t)$. If $\gamma(t)$ had a continuous derivative, then (1.6) would be solvable for all

$$
\rho \in J_{0}=\left(\inf _{t>0} 2 \gamma_{-}^{\prime}(t), \sup _{t>0} 2 \gamma_{+}^{\prime}(t)\right) .
$$

However, $\gamma(t)$ is not necessarily differentiable (cf. [7]). On the other hand, [7] gives sufficient conditions for (1.6) to be solvable with $\rho \in J_{0}$. The argument of [7] involves a mountain pass lemma with an additional condition of the (PS)-type which we avoid here.

The main result of this paper is

Theorem 1.2. Let $g$ satisfy (1.1), (1.2). Then for every $\rho \in J_{0}$ such that (1.6) is not solvable there is a sequence $\rho_{j} \in J_{0}$ and a sequence $u_{j} \in H$, such that

$$
\begin{array}{cc}
\rho_{i}>\rho, & \rho_{j} \rightarrow \rho, \\
\rho_{j} u_{j}=g^{\prime}\left(u_{j}\right), & \left\|u_{j}\right\| \rightarrow \infty .
\end{array}
$$

Theorem 1.2 means that the set of eigenvalues of (1.6) is dense in $J_{0}$ and that a missing eigenvalue always can be approximated by a "blow up" sequence. Theorem 1.2 reflects a technical situation that might be called a mountain impasse. This is the case when a functional has a standard mountain pass geometry but no critical points. This situation is handled by Theorem 2.1 which is a refinement of Schechter's Mountain Pass Alternative [4]. Section 2 contains the proof of Theorem 2.1. Section 3 discusses applications to semilinear elliptic problems. The tangible benefit of Theorem 1.2 is not a mountain impasse itself (which to our best understanding was never observed in elliptic problems), but a relation between solvability and priori bounds, widely used before in the topological approach. We will discuss this in more detail at the end of $\S 3$.

\section{Mountain IMPASSE THEOREM}

Let

$$
G \in C^{1}(H \rightarrow \mathbf{R}), \quad G^{\prime} \in C\left(H_{w} \rightarrow H_{w}\right)
$$

be a weakly lower semicontinuous functional with a mountain pass geometry, as follows. Let $\delta>0, t_{0}>0, e \in H,\|e\|^{2}>t_{0}$ and assume that

$$
G(u) \geq 2 \delta>0 \text { for }\|u\|^{2}=t_{0} \text {, while } G(0), G(e) \leq 0 .
$$

We assume that $G$ has no critical points:

$$
G^{\prime}(u) \neq 0 \quad \text { when } G(u) \geq \delta .
$$


The following condition will also be required:

$$
\begin{aligned}
& \text { if } u_{k} \stackrel{w}{\rightarrow} u_{0}, \lim \sup \left(G^{\prime}\left(u_{k}\right), u_{k}\right) \leq 0 \text { and } \\
& G^{\prime}\left(u_{k}\right)-\left(G^{\prime}\left(u_{k}\right), u_{k}\right) u_{k} /\left\|u_{k}\right\|^{2} \rightarrow 0, \\
& \text { then } u_{k} \rightarrow u_{0} \text { in } H .
\end{aligned}
$$

We should note that this condition of a weak (PS) type becomes a mere weak continuity condition when $G$ is as in (3.2). Let

$$
S_{t}=\left\{u \in H:\|u\|^{2}=t\right\}, \quad B_{t}=\left\{u \in H:\|u\|^{2} \leq t\right\} .
$$

For every $t>\|e\|^{2}$ we define $\Phi(t)$ as a collection of paths $\varphi \in C\left([0,1] \rightarrow B_{t}\right)$ such that

$$
\varphi(0)=0, \quad \varphi(1)=\varepsilon .
$$

Let

$$
\kappa(t)=\inf _{\varphi \in \Phi(t)} \max _{s \in[0,1]} G(\varphi(s)) .
$$

From (2.2) it follows that

$$
\kappa(t) \geq 2 \delta \text { when } t>\|e\|^{2} .
$$

Theorem 2.1. Assume (2.1)-(2.4). There exist a sequence $\alpha_{j}>0, \alpha_{j} \rightarrow 0$, and a sequence $u_{j} \in H \backslash\{0\},\left\|u_{j}\right\| \rightarrow \infty$, such that

$$
\begin{gathered}
G^{\prime}\left(u_{j}\right)=-\alpha_{j} u_{j}, \\
G\left(u_{j}\right) \geq \delta .
\end{gathered}
$$

The proof of Theorem 2.1 will be given as a sequence of lemmas. Relations (2.1)-(2.4) are assumed throughout $\S 2$. The following statement can be found in [5].

Lemma 2.2. Let $Z(u) \in C\left(B_{t} \rightarrow H\right)$ and $Z(u) \neq 0$ on $B_{t} \backslash\{0\}$. Assume that there is a closed subset $Q$ of $B_{t} \backslash\{0\}$ and a $\theta<1$ such that

$$
(Z(u), u)+\theta\|Z(u)\|\|u\| \geq 0, \quad u \in Q .
$$

Then for each $\alpha<(1-\theta)$ there is a locally Lipschitz mapping $Y(u): B_{t} \backslash\{0\} \rightarrow$ $H$ such that

$$
\begin{gathered}
(Z(u), Y(u)) \geq \alpha\|Z(u)\|, \quad u \in B_{t} \backslash\{0\}, \\
(Y(u), u)>0, \quad u \in Q,
\end{gathered}
$$

and

$$
\|Y(u)\| \leq 1, \quad u \in B_{t} \backslash\{0\} .
$$

Lemma 2.3. Assume that there is an $\varepsilon>0$, such that

$$
G^{\prime}(u)=\beta u
$$

has no solution $u$ when

$$
u \in H_{\delta}=\{u \in H: G(u) \geq \delta\},
$$

and $\beta \in[-2 \varepsilon, 0]$. Then for any $t>\|e\|^{2}$ there exist a $\theta<1$ such that

(2.17) $\left(G^{\prime}(u), u\right)+\theta\left\|G^{\prime}(u)\right\|\|u\| \leq 0, \quad u \in H_{\delta} \cap B_{t} \Rightarrow\left(G^{\prime}(u), u\right) \leq-\varepsilon\|u\|^{2}$. 
Proof. Assume the opposite, namely that there is a sequence $u_{j} \in H_{\delta} \cap B_{t}$ and a sequence $\theta_{j} \rightarrow 1, \theta_{j}<1$, such that

$$
\begin{gathered}
\left(G^{\prime}\left(u_{j}\right), u_{j}\right)+\theta_{j}\left\|G^{\prime}\left(u_{j}\right)\right\|\left\|u_{j}\right\| \leq 0, \\
\beta\left(u_{j}\right):=\left(G^{\prime}\left(u_{j}\right), u_{j}\right) /\left\|u_{j}\right\|^{2} \in[-\varepsilon, 0] .
\end{gathered}
$$

Let $u_{i}$ be a renamed weakly convergent subsequence, and $u_{0}=w-\lim u_{j}$. By (2.1) $G^{\prime}\left(u_{j}\right) \stackrel{w}{\rightarrow} G^{\prime}\left(u_{0}\right)$ and therefore $G^{\prime}\left(u_{j}\right)$ is bounded in norm. Then (2.18) easily implies

$$
G^{\prime}\left(u_{j}\right)-\beta\left(u_{j}\right) u_{j} \rightarrow 0 .
$$

Then by $(2.4) u_{j} \rightarrow u_{0}$ in $H, G\left(u_{0}\right) \geq \delta$, and

$$
G^{\prime}\left(u_{0}\right)=\beta\left(u_{0}\right) u_{0}, \quad u_{0} \in H_{\delta} \cap B_{t} .
$$

By (2.19) $\beta\left(u_{0}\right) \in[-\varepsilon, 0]$ which contradicts assumptions of the lemma.

Lemma 2.4. There is a number $r(t)>0$ and a number $\mu>0$ independent of $t$ such that

$$
\begin{gathered}
\left\|G^{\prime}(u)\right\| \geq r(t), \quad u \in H_{\delta} \cap B_{t}, \\
\|u\| \geq 2 \mu, \quad u \in H_{\delta} \cap B_{t} .
\end{gathered}
$$

Proof. (1) Assume that (2.22) fails. Then there is a sequence $u_{j} \stackrel{w}{\rightarrow} u_{0} \in B_{t}$, such that $G^{\prime}\left(u_{j}\right) \rightarrow 0$. Then by (2.4) $u_{j} \rightarrow u_{0}$ in $H, u_{0} \in H_{\delta} \cap B_{t}$, and $G^{\prime}\left(u_{0}\right)=0$, which contradicts $(2.2)$.

(2) Consider the lower bound of $\|u\|$ on $H_{\delta}$. If $u_{j} \rightarrow 0$ on $H_{\delta}$, then $G\left(u_{j}\right) \rightarrow G(0) \leq 0$ by (2.2), which contradicts the assumption on $u_{j}$.

Lemma 2.5. Under assumptions of Lemma 2.3,

$$
D_{t}^{+} \kappa(t) \leq-\frac{1}{2} \varepsilon \mu^{2} / t, \quad t>\|e\|^{2},
$$

with $\mu$ as in (2.23).

Proof. (1) Let us define the following sets:

$$
\begin{aligned}
& Q_{0}=\left\{u \in B_{2 t}:|G(u)-\kappa(t)| \leq \delta / 2\right\}, \\
& \widetilde{Q}_{0}=\left\{u \in B_{2 t}:|G(u)-\kappa(t)| \geq \delta\right\}, \\
& Q_{1}=\left\{u \in B_{2 t}: \frac{\left(G^{\prime}(u), u\right)}{\left\|G^{\prime}(u)\right\|\|u\|} \leq-1+\eta\right\}, \\
& Q_{2}=\left\{u \in B_{2 t}: \frac{\left(G^{\prime}(u) u\right)}{\left\|G^{\prime}(u)\right\|\|u\|} \geq-1+2 \eta\right\},
\end{aligned}
$$

where $t>\|e\|^{2}, 0<2 \eta<1-\theta$ with $\theta$ as in Lemma 2.3 applied to the ball $B_{2 t}$. Let

$$
\begin{aligned}
& \chi_{0}(u)=d\left(u, \widetilde{Q}_{0}\right) /\left(d\left(u, Q_{0}\right)+d\left(u, \widetilde{Q}_{0}\right)\right), \\
& \chi_{1}(u)=d\left(u, Q_{2}\right) /\left(d\left(u, Q_{1}\right)+d\left(u, Q_{2}\right)\right), \\
& \chi_{2}(u)=1-\chi_{1}(u), \quad u \in H .
\end{aligned}
$$

The functions (2.26) are Lipschitz continuous, their range is $[0,1]$, they equal one on $Q_{0}, Q_{1}, Q_{2}$, respectively, and vanish, respectively, on $\widetilde{Q}_{0}, Q_{2}$ and $Q_{1}$. 
We now wish to apply Lemma 2.2 for $B_{2 t}$ with $Z=G^{\prime}, Q=\operatorname{supp} \chi_{1} \cap$ supp $\chi_{0}=\overline{B_{2 t} \backslash\left\{\widetilde{Q}_{0} \cup Q_{2}\right\}}$ and $\theta$ as in Lemma 2.3. Consider the initial value problem

$$
\begin{gathered}
d \sigma / d h=\chi_{0}(\sigma) \chi_{1}(\sigma) \sigma-N \chi_{0}(\sigma) \chi_{2}(\sigma) Y(\sigma) /\|Y(\sigma)\|, \\
\left.\sigma(h)\right|_{h=0}=\varphi, \quad \varphi \in H, \quad N=2 \varepsilon t /(1-2 \eta) r
\end{gathered}
$$

The right-hand side in (2.27) is locally Lipschitz continuous in $\sigma$ and thus the problem has a unique $C^{1}$-solution $\sigma$ defined for all $h$. Note that if $\varphi=0$ or $\varphi=e$, then so is $\sigma$ for all $h$.

(2) Let $\varphi_{j} \in \Phi(t)$ be a minimizing sequence for (2.7) and $\sigma_{j}$ be correspondent solutions of (2.27)-(2.28). Then for $t_{1} \in(t, 2 t)$,

$$
\kappa\left(t_{1}\right) \leq \sup _{s \in[0,1]} G\left(\sigma_{j}(h ; s)\right)
$$

as long as

$$
\left\|\sigma_{j}(h ; s)\right\|^{2}<t_{1} \quad \text { for all } s \in[0,1] .
$$

Let us establish a bound on $h$ that implies (2.30). By (2.27)

$$
\frac{d}{d h}\left\|\sigma_{j}\right\|^{2}=2 \chi_{0} \chi_{1}\left\|\sigma_{j}\right\|^{2}-2 \chi_{0} \chi_{2} N\left(Y\left(\sigma_{j}\right), \sigma_{j}\right)\left\|Y\left(\sigma_{j}\right)\right\|^{-1} \leq 2\left\|\sigma_{j}\right\|^{2} .
$$

Therefore,

$$
\left\|\sigma_{j}(h ; s)\right\|^{2} \leq t e^{2 h}
$$

Consequently, assuming

$$
0 \leq h \leq \frac{1}{2} \ln \left(t_{1} / t\right)
$$

we obtain (2.30). In the further course of the proof $h$ will be subject to additional bounds from above.

(3) Let us estimate the derivative of $G(\sigma)$.

$$
\frac{d}{d h} G\left(\sigma_{j}\right)=\chi_{0} \chi_{1}\left(G^{\prime}\left(\sigma_{j}\right), \sigma_{j}\right)-N \chi_{0} \chi_{2}\left(G^{\prime}\left(\sigma_{j}\right), Y\left(\sigma_{j}\right)\right) /\left\|Y\left(\sigma_{j}\right)\right\| \text {. }
$$

By Lemma 2.2 as already applied,

$$
\left(G_{j}^{\prime}(\sigma), Y\left(\sigma_{j}\right)\right) /\left\|Y\left(\sigma_{j}\right)\right\| \geq(1-2 \eta)\left\|G^{\prime}\left(\sigma_{j}\right)\right\| \quad \text { when } \sigma \in \operatorname{supp} \chi_{0} \chi_{2} .
$$

By Lemma 2.3

$$
\left(G^{\prime}\left(\sigma_{j}\right), \sigma_{j}\right) \leq-\varepsilon\left\|\sigma_{j}\right\|^{2} \quad \text { when } \sigma \in \operatorname{supp} \chi_{0} \chi_{1} .
$$

Then (2.34) yields

$$
\begin{aligned}
\frac{d}{d h} G\left(\sigma_{j}\right) & \leq-\varepsilon \chi_{0} \chi_{1}\left\|\sigma_{j}\right\|^{2}-(1-2 \eta) N \chi_{0} \chi_{2}\left\|G^{\prime}\left(\sigma_{j}\right)\right\| \\
& \leq-\varepsilon \chi_{0} \chi_{1}\left\|\sigma_{j}\right\|^{2}-(1-2 \eta) N \chi_{0} \chi_{2} r\left\|\sigma_{j}\right\|^{2} / 2 t \\
& \leq-\varepsilon \chi_{0}\left(\chi_{1}+\chi_{2}\right)\left\|\sigma_{j}\right\|^{2}=-\varepsilon \chi_{0}\left(\sigma_{j}\right)\left\|\sigma_{j}\right\|^{2}
\end{aligned}
$$

(4) Consider the following sets of $s \in[0,1]$. Let

$$
I_{1}=\{s \in[0,1]:|G(\varphi(s))-\kappa(t)| \geq \delta / 2\} .
$$


For $j$ large enough the inequality in (2.38) holds only if $G\left(\varphi_{j}(s)\right) \leq \kappa(t)-\delta / 2$, since $\varphi_{j}$ is a minimizing sequence and $\kappa(t)$ is approximated by the maximal values of $G\left(\varphi_{j}(s)\right)$. By $(2.37), G\left(\sigma_{j}(h ; s)\right) \leq \kappa(t)-\delta / 2$ for $s \in I_{1}$. Now let $I_{2}$ be a subset of $[0,1] \backslash I_{1}$, such that $\sigma_{j}(h ; s) \in Q_{0}$ for all $h \in\left[0, h_{1}\right]$, $h_{1}:=\frac{1}{2} \ln \left(t_{1} / t\right)$, and $I_{3}=[0,1] \backslash\left(I_{1} \cup I_{2}\right)$. On $I_{2}$ (2.37) implies

$$
\frac{d}{d h} G\left(\sigma_{j}\right) \leq-\varepsilon\left\|\sigma_{j}\right\|^{2} \text {. }
$$

By (2.31)

$$
\frac{d}{d h}\left\|\sigma_{j}\right\|^{2} \geq-2 N\left\|\sigma_{j}\right\|
$$

and consequently,

$$
\left\|\sigma_{j}\right\| \geq\left\|\varphi_{j}\right\|-N h, \quad s \in I_{2} .
$$

Since $\varphi_{j}(s) \in H_{\delta}$ when $s \in I_{2}$, Lemma 2.4 implies

$$
\left\|\sigma_{j}\right\| \geq 2 \mu-N h
$$

and assuming

$$
h \leq \mu / N
$$

one has

$$
\left\|\sigma_{j}\right\| \geq \mu \text { for } s \in I_{2},
$$

and therefore,

$$
\frac{d}{d h} G\left(\sigma_{j}\right) \leq-\varepsilon \mu^{2}, \quad s \in I_{2} .
$$

Finally, if $s \in I_{3}$, let $h_{0} \in\left[0, h_{1}\right]$ be a maximal $h$, such that $\sigma_{j}(h ; s) \in Q_{0}$ for $h \in\left[0, h_{0}\right]$. Then

$$
G\left(\sigma_{j}(h ; s)\right) \leq G\left(\sigma_{j}\left(h_{0}, s\right)\right)=\kappa(t)-\delta / 2, \quad s \in I_{3} .
$$

Combining (2.38), (2.45), and (2.44), one has from (2.29) that

$$
\begin{aligned}
\kappa\left(t_{1}\right) & \leq \max _{s \in[0,1]} G\left(\sigma_{j}(h ; s)\right) \\
& \leq \max \left\{\kappa(t)-\delta / 2, \max _{s \in I_{3}} G\left(\varphi_{j}\right)-\varepsilon \mu^{2} h\right\} \\
& \leq \max \left\{\kappa(t)-\delta / 2, m_{j}-\varepsilon \mu^{2} h\right\},
\end{aligned}
$$

where $m_{j}=\max _{s \in[0,1]} G\left(\varphi_{j}\right), m_{j} \rightarrow \kappa(t)$. With $j$ large enough and an additional upper bound on $h$

$$
h<\delta / 6 \varepsilon \mu^{2}
$$

one has

$$
\kappa\left(t_{1}\right) \leq \kappa(t)-\varepsilon \mu^{2} h .
$$

Relation (2.49) is valid only as far as $h$ satisfies restrictions (2.48), (2.43), and (2.33). Three of them can be reduced to (2.33) when

$$
t_{1}<\min \left\{2 t, t e^{2 h_{2}}, t e^{2 h_{3}}\right\}, \quad \text { where } h_{2}=\frac{\mu}{N} \text { and } h_{3}=\frac{\delta}{6 \varepsilon \mu^{2}} .
$$


Then (2.49) with $h=\frac{1}{2} \ln \left(t_{1} / t\right)$ immediately implies (2.24).

Proof of Theorem 2.1. It is already proved in [4] that for any $t>\|\varepsilon\|^{2}$ there is a $u \in H_{\delta} \cap B_{t}$ and $\alpha>0$ such that

$$
G^{\prime}(u)=-\alpha u \text {. }
$$

Assume that there are no sequences $\alpha_{j}>0, u_{j} \in H_{\delta}$ satisfying (2.51) and such that $\alpha_{j} \rightarrow 0$. Then the conditions of Lemma 2.3 are satisfied with some $\varepsilon>0$ and by Lemma 2.5

$$
\limsup _{t \rightarrow \infty} \kappa(t) \rightarrow-\infty
$$

which contradicts (2.8). Therefore $\left\{\alpha_{j}\right\}$ necessarily has a subsequence with zero limit. Assume now that $\left\{u_{j}\right\}$ has a bounded subsequence. Then there is a weakly convergent renamed subsequence $u_{j} \stackrel{w}{\rightarrow} u_{0}$ and

$$
G^{\prime}\left(u_{j}\right) \rightarrow 0 \text {. }
$$

Then by (2.4) $u_{j} \rightarrow u_{0}, G\left(u_{0}\right) \geq \delta$, and $G^{\prime}\left(u_{0}\right)=0$, which contradicts (2.3). Thus $\left\|u_{j}\right\| \rightarrow \infty$.

\section{APPLICATIONS TO ELLIPTIC PROBLEMS}

We wish to show now that Theorem 2.1 implies Theorem 1.2. Our argument is somewhat repetitious of [7] and we omit details.

(1) Let

$$
\rho_{0} \in J_{0}
$$

and

$$
G(u)=\frac{1}{2} \rho_{0}\|u\|^{2}-g(u)+c, \quad c \leq g(0) .
$$

It is easy to see that if $g$ satisfies (1.1) and (1.2), then $G$ satisfies (2.1).

(2) Let us verify (2.2). Let

$$
\Gamma(t)=\frac{1}{2} \rho_{0} t-\gamma(t)+c .
$$

If the function $\Gamma(t)$ has a point of a local minimum on $(0, \infty)$, then $G$ has a nonzero critical point (cf. [6] or [7]), which contradicts the assumptions. If the function $\Gamma(t)$ is monotone, then by Theorem 1.2 all the derivatives of $\gamma(t)$ must be either greater or smaller than $\frac{1}{2} \rho_{0}$ which contradicts (3.1).

The remaining possibility is that $\Gamma(t)$ has a global maximum at $t=t_{0} \epsilon$ $(0, \infty)$. Then (2.2) is satisfied with the following choices. Let $t_{1}>t_{0}$ and let $e$ be an element of a maximizing sequence for $g$ on $S_{t_{1}}$, such that

$$
\frac{1}{2} \rho_{0} t_{1}-g(e)+c<M+c,
$$

where

$$
M=\frac{1}{2} \rho_{0} t_{0}-\gamma\left(t_{0}\right)
$$

Finally, set

$$
\delta=\frac{1}{2} \min \left(M-\frac{1}{2} \rho_{0}\|e\|^{2}+g(e), M+g(0)\right)
$$

and

$$
c=2 \delta-M .
$$


If one assumes that (1.6) has no solution with $\rho=\rho_{0}$, then $G$ satisfies (2.3). Now note that (2.4) follows from (1.2) and one can apply Theorem 2.1.

We discuss here only the applications to the problem

$$
-\rho \Delta u=f(u), \quad u \in H_{0}^{1}(\Omega) \backslash\{0\},
$$

where $\Omega \subset \mathbf{R}^{n}, n \geq 3$, is an open bounded set, $f: \mathbf{R} \rightarrow \mathbf{R}$ is continuous and subcritical at infinity, i.e.,

$$
f(s)=o\left(|s|^{(n+2) /(n-2)}\right) \quad \text { as } s \rightarrow \infty .
$$

Let

$$
\begin{aligned}
& F(s)=\int_{0}^{s} f(\sigma) d \sigma, \\
& g(u)=\int_{\Omega} F(u) d x .
\end{aligned}
$$

It is well known that $g$ satisfies (1.1) and (1.2) on $H_{0}^{1}(\Omega)$. Equation (3.8) is an equation for a critical point of $g$ on

$$
S_{t}=\left\{u \in H_{0}^{1}(\Omega): \int_{\Omega}|\nabla u|^{2}=t\right\} .
$$

Theorem 1.2 then implies that (3.8) is solvable for a dense subset of $\rho \in J_{0}$. The interval $J_{0}$ is defined here as an open interval between the lower and upper bound of the slopes on the graph of

$$
2 \gamma(t)=\sup _{u \in S_{t}} 2 \int_{\Omega} F(u) d x .
$$

One can reverse Theorem 1.2 and state the solvability of (1.6) for all $\rho \in J_{0}$ with an additional condition of an a priori bound on a Hilbert norm of $u$.

Theorem 3.1. Assume (1.1), (1.2), and (1.8). If there is $a \nu>0$ and $a c>0$, such that for any $u$ satisfying (1.6) with $\left|\rho-\rho_{0}\right|<\nu$,

$$
\|u\| \leq c,
$$

then (1.6) has a solution with $\rho=\rho_{0}$.

This statement is an elementary corollary of Theorem 1.2.

There are several important results which establish a priori bounds in $L^{\infty}$ motivated by the topological approach to (3.8) (cf. [3] and references therein). The following statement uses an argument from [3].

Corollary 3.2. Let $n \geq 3, \Omega$ be starshaped, $f \geq 0$, and let

$$
\frac{F(s)}{s^{\sigma}} \text { be a decreasing function near } s=+\infty \text { with some } \sigma<\frac{2 n}{n-2} \text {. }
$$

Then the problem (3.8) satisfies the condition (3.14) and consequently is solvable for all $\rho \in J_{0}$.

Proof. Since $\Omega$ is starshaped, a well-known Pohozhaev-Rellich identity (resulting from multiplication of $(3.8)$ by $(x \cdot \nabla) u)$ provides

$$
\rho\|u\|^{2} \leq \frac{2 n}{n-2} \int F(u) .
$$


At the same time, multiplication of (3.8) by $u$ gives

$$
\rho\|u\|^{2}=\int f(u) u .
$$

From (3.15) and since the problem might have only positive solutions, one has

$$
\int F(u) \leq \sigma \int f(u) u+c, \quad c \in \mathbf{R} .
$$

Then (3.16)-(3.18) immediately provide (3.14).

This corollary includes cases of $f$ with sub- (or super-)linear behavior both at 0 and at $\infty$.

\section{REFERENCES}

1. M. S. Berger, Nonlinearity and functional analysis, Academic Press, New York, 1977.

2. F. E. Browder, Infinite dimensional manifolds and nonlinear elliptic eigenvalue problems, Ann of Math. 82 (1965), 459-477.

3. G. D. de Figueiredo, P.-L. Lions, and R. Nussbaum, A priori estimates and existence of positive solutions of semilinear elliptic equations, J. Math. Pures Appl. 61 (1962), 41-63.

4. M. Schechter, Mountain pass alternative, Adv. in Appl. Math. 12 (1991), 91-105.

5. __ The Hampwile theorem for nonlinear eigenvalues, Duke Math. J. 59 (1989), 325-335.

6. M. Schechter and K. Tintarev, Points of spherical maxima and solvability of semilinear elliptic problems, Canad. J. Math. 43 (1991), 1-7.

7. M. Schecter and K. Tintarev, Eigenvalues for semilinear boundary value problems, Arch. Rational Mech. Anal. 113 (1991), 197-208.

Department of Mathematics, University of California, Irvine, California 92717

E-mail address: tintarev@math.uci.edu 